
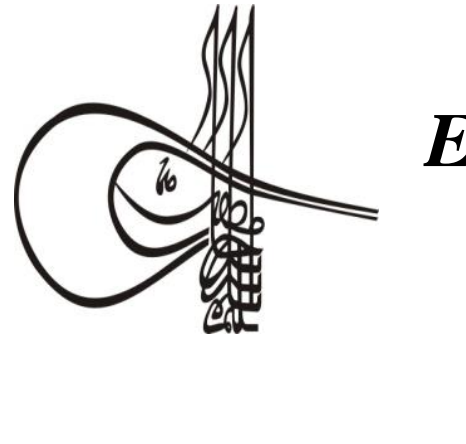

Received/Geliş: 14.06 .2019

Go Report Dates/Rapor Tarihleri: Referee 1 (22.07.2019)-Referee 2 (23.07.2019)

This article was checked by iThenticate.
Turkigh Studies

Economics, Finance, Politics

Volume 14 Issue 3, 2019, p. 1035-1043

DOI: 10.29228/TurkishStudies.23329

ISSN: 2667-5625

Skopje/MACEDONIA-Ankara/TURKEY

Research Article / Araştırma Makalesi

Article Info/Makale Bilgisi

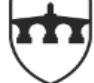

INTERNATIONAL BALKAN UNIVERSITY

EXCELLENCE FOR THE FUTURE IBU.EDU.MK

$\checkmark$ Accepted/Kabul: 10.09 .2019

\title{
DOĞRUDAN YABANCI YATIRIMLARIN ÜLKELERINN EKONOMIKK PERFORMANSLARINA ETKİLERİ: MINT ÜLKELERİ ÜZERİNE TARİHSEL BİR ANALİZ
}

\author{
Seçil ŞENEL UZUNKAYA*
}

\begin{abstract}
öz
$\mathrm{Bu}$ çalışmanın amacı doğrudan yabancı yatırımların ülkelerin ekonomik büyümeleri üzerine olan etkilerinin araștırılmasıdır. Söz konusu amaca ulaşabilmek için MINT ülkeleri (Meksika, Endonezya, Nijerya ve Türkiye) inceleme kapsamına alınmıștır. Doğrudan yabancı yatırımlar özellikle gelişmekte olan ülke ekonomileri için önem arz etmektedir. Bahsi geçen ülkeler gelişmiş ülke statüsüne ulaşabilmek için yatırımları arttırmayı amaç edinmişlerdir. Analiz sürecinde Pedroni eşbütünleşme analizi kullanılmıştır. Ayrıca, 1974 ve 2017 dönem aralığındaki yıllık veriler kullanılarak tarihsel bir analiz yapılması hedeflenmiştir. Dönem aralığının uzun alınmasından dolayı tarihsel bir analiz yapılabilmesi mümkün olmuştur. Dolayısıyla, bu çalışmanın iki farklı özgünlügünden bahsedebilmek mümkündür. İlk olarak, analiz MINT ülkelerinin kalkınmaları adına yol gösterici niteliğe sahiptir. Öte yandan, Pedroni panel eşbütünleşme analizi bu konu başlığı altında ilk defa dikkate alınmaktadır. MİNT ülkelerinin seçilmesinin temel nedeni ilgili ülkelerin gelişmekte olan ülkeler grubunda olmasına karşın özellikle coğrafi konumlarından dolayı büyüme potansiyelinin bulunmasıdır. Netice itibarıyla, MINT ülkeleri için doğrudan yabancı yatııımlar ve ekonomik büyüme arasında uzun süreli ilişki olduğu sonucuna varılmıştır. Dolayısıyla, MINT ülkelerinde hükümetlerin doğrudan yabancı yatırımı arttırabilmek adına vergi indirimi ve yer desteği gibi teşvikleri vermeleri yerinde olacaktır. Bu sayede, doğrudan yabancı yatırımların arttırılabilmesi mümkün olabilecektir. Belirtilen bu durum söz konusu ülkelerin gelişmiş ülke seviyesine ulaşabilmelerinde kolaylık sağlayacaktır.
\end{abstract}


Anahtar Kelimeler: Doğrudan Yabanc1 Yatırım, Ekonomik Büyüme, İktisat Tarihi, Pedroni Panel Eşbütünleşme Analizi

\title{
THE EFFECTS OF FOREIGN DIRECT INVESTMENTS ON THE ECONOMIC PERFORMANCE: A HISTORICAL ANALYSIS ON THE MINT COUNTRIES
}

\begin{abstract}
The aim of this study is to investigate the effects of foreign direct investments on the economic growth of countries. MINT countries (Mexico, Indonesia, Nigeria and Turkey) are selected in order to achieve this objective of the study. Foreign direct investments are especially important for the economies of developing countries. These countries have aimed to increase investments in order to reach the status of developed countries. Pedroni cointegration analysis was used in the analysis process. Furthermore, it is aimed to make a historical analysis by using the annual data in the period between 1974 and 2017. Due to the long period interval, a historical analysis is possible. Therefore, it is possible to mention two different specificities of this study. First, the analysis is a guide for the development of MINT countries. On the other hand, Pedroni panel cointegration analysis is considered for the first time under this topic. The main reason for the selection of MINT countries is that although the countries concerned are in the group of developing countries, there is a potential for growth especially due to their geographical location. As a result, it was concluded that there is a longterm relationship between direct foreign investments and economic growth for the MINT countries. Therefore, it would be appropriate for governments in MINT countries to provide incentives such as tax reduction and location support to increase foreign direct investment. This will help the countries in question to reach the developed country level.
\end{abstract}

\section{STRUCTURED ABSTRACT}

Foreign investments are divided into two different classes as portfolio investments and foreign direct investments. In portfolio investments, investors invest in capital market instruments such as stocks and bonds in the country. Foreign direct investments represent the investments made in another country for production. These investments can be in the form of acquiring a company or becoming a partner to that company, or opening a new firm is also a part of the foreign investment class. Unlike portfolio investments, foreign direct investments are not expected to emerge suddenly. Therefore, it is expressed as a more permanent and permanent investment type. It is possible to talk about the many advantages of foreign direct investments. First of all, these new investments will increase the production capacity in the country. This situation will also contribute to the economic growth of the country and the reduction of unemployment. In addition, the entry of new firms into the market will increase competition in the country. This will help to decrease prices and increase quality. The aim of this study is to investigate the effects of foreign direct investments on the

Turkish Studies - Economics, Finance, Politics

Volume 14 Issue 3, 2019 
economic growth of countries, which have many advantages as described above. MINT countries to achieve this goal (Mexico, Indonesia, Nigeria and Turkey) were included in the study. In this study, annual data between 1974 and 2017 period are taken into consideration. This data was obtained from the World Bank website. Due to the long period interval, a historical analysis is possible. Additionally, Mexico, Indonesia, Nigeria and Turkey were examined countries, consisting of MINT. The main reason for the selection of these countries is the fact that although the countries concerned are in the group of developing countries, there is a potential for growth especially due to their geographical location. In this study, it is examined whether foreign direct investments affect the economic development of countries. In this context, Mexico, Indonesia, Nigeria and Turkey consists of the MINT countries were included in the study.

Pedroni panel cointegration analysis was used in the analysis process of the study. There are two different stages in the analysis of the study. In the first stage, the stationarity tests of the variables were performed. In the second stage, Pedroni panel cointegration test was applied. In addition, it is aimed to make a historical analysis by using annual data between 1974 and 2017 period. The issue of foreign direct investment has been addressed by many authors in the literature for different purposes. Some of the studies have examined issues affecting the amount of foreign direct investment in countries. The analysis of the determinants of foreign direct investment in OECD and developing countries revealed that market size is the most important factor affecting foreign investment.

In another study in which developing countries were included in the study, it was emphasized that factors such as crime rate, employee education level and site selection were important in foreign direct investment decisions. The study examines the issues affecting foreign direct investments in European countries and determined that the policies of governments have an impact on foreign investors.

It has been concluded that many different variables such as technological development have an impact on the decisions of foreign investors. In another study examining European countries, they stated that industrial power in the country is important in the preference of foreign investors. In the research conducted on the African Union, it is concluded that the inflation rate affects foreign direct investments. In the research conducted for ASEAN countries, it was concluded that the size of the market in these countries is the most important factor affecting foreign direct investments. For the same country groups, variables such as market risk and effective legal system were found to be effective. Matters affecting foreign direct investments in Turkey were also discussed by different researchers. With MARS method in studies have investigated factors affecting Turkey's foreign direct investment. As a result, it is concluded that the high current account deficit affects foreign investments negatively. It was also concluded that the salaries of workers in the country are important. In addition to the factors affecting foreign direct investment, the relationship between foreign direct investment and economic growth has been the subject of many studies. According to the results of the analysis, it was concluded that foreign investments positively affected the economic growth of the countries.

Turkish Studies - Economics, Finance, Politics

Volume 14 Issue 3, 2019 
Pedroni panel cointegration analysis is used to test the presence of long-term relationship between variables. During the analysis process, 7 different tests (Panel v-Statistic, Panel rh-Statistic, Panel PP-Statistic, Group AD-Statistic, Group r-Statistic, Group PP-Statistic and Group ADF-Statistic) are performed by the system. If at least 4 of these tests are meaningful, it is possible to talk about the long-term relationship between the variables. As a result, it is concluded that there is a longterm relationship between foreign direct investment and economic growth for MINT countries. In other words, increasing foreign direct investment contributes to the economic development of these countries. Therefore, it would be appropriate for governments in MINT countries to give incentives such as tax reduction and location support in order to increase foreign direct investment. In this way, it will be easier for these developing countries to reach the level of developed countries. This issue, which is important in the literature, is tested on MINT countries and aimed to contribute. However, it will be appropriate to apply this issue which is important in future studies to different country groups. On the other hand, it is thought that the use of different methods such as panel regression, Engle Granger cointegration analysis and Dumitrescu Hurlin panel causality analysis will contribute to these studies.

Keywords: Foreign Direct Investment, Economic Growth, Economic History, Pedroni Panel Cointegration Analysis.

\section{Giriş}

Yabancı yatırımlar, portföy yatırımları ve doğrudan yabancı yatırımlar olmak üzere iki farklı sınıfa ayrılmaktadır. Portföy yatırımlarında yatırımcılar ülkedeki hisse senedi ve tahvil gibi sermaye piyasası araçlarına yatırım yapmaktadırlar. Dolayısıyla, bahsi geçen bu yatırımlarından faiz ve kar payı geliri elde edilmesi hedeflenmektedir. Belirtilen bu tanımdan da anlaşlabileceği gibi ülke ekonomisi için önemli olan portföy yatırımlarının ülkeden ani bir şekilde çıkma riski de söz konusudur (Borensztein vd. 1998:115).

Doğrudan yabancı yatırımlar ise başka bir ülkede üretime yönelik olarak yapılan yatırımları ifade etmektedir. Belirtilen bu yatırımlar bir firmayı satın almak veya o firmaya ortak olmak şeklinde olabileceği gibi, yeni bir firma açmak da doğrudan yabancı yatırım sınıfına girmektedir. Portföy yatırımlarının aksine doğrudan yabancı yatırımların ülkeden ani bir şekilde çıkması beklenmemektedir. $\mathrm{Bu}$ yüzden daha sürekli ve kalıcı yatırım türü olarak ifade edilmektedir.

Doğrudan yabancı yatırımların birçok avantajlarından söz edebilmek mümkünüdür. İlk olarak, yapılan bu yeni yatırımlar sayesinde ülkedeki üretim kapasitesi artacaktır. Söz konusu durum ayrıca ülkenin ekonomik anlamda büyümesine ve işsizliğin azaltılmasına katkı sağlayacaktır. Ek olarak, yeni firmaların pazara girmesi ülkedeki rekabeti arttıracaktır. Bu durum da hem fiyatların azalmasına hem de kalitenin artmasına yardımcı olacaktır. Belirtilen bu olumlu yanlara karşı, doğrudan yabancı yatırımların birtakım dezavantajları da bulunmaktadır. Örnek olarak, ülkedeki yerel firmalar, yatırım yapan büyük ölçekli firmalar ile rekabet edemeyerek faaliyetlerine son verebilirler. Bu durum da ülke ekonomisini olumsuz olarak etkileyecektir.

Doğrudan yabancı yatırım özellikle gelişmekte olan ülke ekonomileri için önem arz etmektedir. Bahsi geçen ülkeler gelişmiş ülke statüsüne ulaşabilmek için yatırımları arttırmayı amaçlamaktadırlar (Tunay ve Yüksel, 2017:1627). Bu hedefe ulaşabilmek için de ülkelerin çoğu doğrudan yabancı yatırımların ülkelerine gelebilmesi için vergi avantajı, yer tahsisi gibi birtakım teşvikler sunmaktadır. Bu yatırımlar ile hem ülkenin üretim seviyesini arttırmayı hem de işsizliği azaltmayı hedeflemektedirler.

Turkish Studies - Economics, Finance, Politics

Volume 14 Issue 3, 2019 
Öte yandan, ilgili yatırımlar sayesinde ülkenin gayri safi milli hasılasının da artması söz konusu olabilmektedir.

Bu çalışmada doğrudan yabancı yatırımlarının ülkelerin ekonomik büyümelerine etkileri analiz edilmektedir. Meksika, Endonezya, Nijerya ve Türkiye'den oluşan MINT ülkeleri analiz kapsamına alınmıştır. Ayrıca, Pedroni panel eşbütünleşme analizi kullanılarak sonuca ulaşılmaya çalışılmıştır. Dolayısıyla, bu çalışmanın iki farklı özgünlüğünden bahsedebilmek mümkündür. İlk olarak, analiz MINT ülkelerinin kalkınmaları adına yol gösterici niteliğe sahiptir. Öte yandan, Pedroni panel eşbütünleşme analizi bu konu başlığı altında ilk defa dikkate alınmaktadır.

\section{Teorik Literatür}

Doğrudan yabancı yatırım konusu literatürde farklı amaçlarla çok sayıda yazar tarafindan ele alınmıştır. Çalışmaların bazıları ülkelerdeki doğrudan yabancı yatırım miktarına etki eden hususları incelemiştir. Economou vd. (2017:527) çalışmasında OECD ve gelişmekte olan ülkelerdeki doğrudan yabancı yatırımın belirleyicilerini analiz etmişlerdir. Sonuç olarak, piyasanın büyük olmasının yabancı yatırımları en çok etkileyen hususlar olduğu anlaşılmıştır. Lucke ve Eichler (2016:935), Dinçer vd. (2019a:245), Bekhet ve Al-Smadi (2015:27), Tokunaga ve Iwasaki (2017:2771), Adalı ve Yüksel (2017:109) ve Nielsen vd. (2017:62) de gelişmekte olan ülkeleri inceleme kapsamına almış ve suç oranı, çalışanların eğitim düzeyi ve yer seçimi gibi faktörlerin doğrudan yabancı yatırım kararlarında önemli olduğunun altını çizmiştir.

Castellani vd. (2016:671) Avrupa ülkelerindeki doğrudan yabancı yatırımlara etki eden hususları incelemiştir. Yapılan analiz sonucunda hükümetlerin politikalarının yabancı yatırımcılar üzerinde etkili olduğu belirlenmiştir. Villaverde ve Maza (2015:209) da çalışmalarında söz konusu ilişkiyi aynı bölge ülkeleri üzerinde incelemiştir. Netice itibarıyla, teknolojik gelişme gibi birçok farklı değişkenlerin yabancı yatırımcıların kararları üzerinde etkili olduğu sonucuna varılmıştır. Jimborean ve Kelber (2017:25), Dinçer, Yüksel ve Martinez (2019:152), Jude ve Silaghi (2016:39), Dinçer vd. (2019b:296), Tang (2017:77) ve Young (2017:141) çalışmalarında Avrupa ülkelerini inceleme kapsamına almış ve ülkedeki sanayi gücünün yabancı yatırımcıların tercihinde önemli olduğunu belirtmişlerdir.

Kariuki (2015:346) aynı konuyu Afrika Birliği üzerinde incelemiştir. Enflasyon oranının doğrudan yabancı yatırımları etkilediği sonucuna ulaşılmıştır. Öte yandan, Xaypanya vd. (2015:243) ise belirtilen bu konuyu ASEAN ülkeleri için incelemiştir. Ülkelerdeki pazarın büyüklüğünün doğrudan yabanc1 yatırımları etkileyen en önemli faktör olduğu sonucuna varılmıştır. Benzer şekilde, Anuchitworawong ve Thampanishvong (2015:318), Lv ve Spigarelli (2016:339), Cassidy (2017:42) ve Erdogan ve Unver (2015:82) de aynı ülke grupları için piyasa riski, etkin hukuk sistemi gibi değişkenlerin etkili olduğunu vurgulamışlardır.

Türkiye'deki doğrudan yabancı yatırımı etkileyen hususlar da farklı araştırmacılar tarafından ele alınmıştır. Zengin vd. (2018:1309) çalışmasında MARS yöntemi ile Türkiye'nin doğrudan yabancı yatırımına etki eden faktörleri araştırmışlardır. Sonuç olarak, cari işlemler açığının yüksek olmasının yabancı yatırımları olumsuz etkilediği sonucuna varılmıştır. Bu çalışmanın yanı sıra, Seker vd. (2015:347), Aybar (2016:79), Arun ve Y1ldırım (2017:226) ve Cergibozan ve Demir (2017:227) de Türkiye'yi inceleme kapsamına almıştır. Söz konusu çalışmalarda ülkedeki işçi maaşlarının önemli olduğu sonucuna ulaşılmıştır.

Doğrudan yabancı yatırımları etkileyen faktörlere ek olarak, doğrudan yabancı yatırımlar ve ekonomik büyüme arasındaki ilişki de birçok çalışmaya konu olmuştur. Iamsiraroj (2016:116) çalışmasında 124 farklı ülkeyi inceleme kapsamına almıştır. Analiz sonuçlarına göre yabancı yatırımların ülkelerin ekonomik büyümesini olumlu etkilediği sonucuna varmıştır. Iamsiraroj ve Ulubaşoğlu (2015:200), Salahuddin vd. (2018:2002), Paramati vd. (2016:29), Solarin ve Shahbaz 
(2015:835), Su ve Liu (2016:97) ve Sunde (2017:434) de çalışmalarında benzer sonucu vurgulamışlardır. Öte yandan, Seyoum vd. (2015:45) çalışmasında böyle bir ilişkinin bazı ülkeler için geçerli olmadığını vurgulamıştır. Söz konusu çalışmaların incelenmesi neticesinde doğrudan yabancı yatırım konusu ile ilgili olarak farklı bir yöntem kullanarak yapılacak yeni bir çalışmaya ihtiyaç olduğu anlaşılmaktadır.

\section{MINT Ülkeleri Üzerine Bir Uygulama}

\subsection{Veri Seti ve Ülkeler}

Çalışmada 1974 ve 2017 dönem aralığındaki yıllık veriler dikkate alınmıştır. Bu veriler Dünya Bankası'na ait internet sitesinden temin edilmiştir. Dönem aralığının uzun alınmasından dolayı tarihsel bir analiz yapılabilmesi mümkün olmuştur. Ayrıca, Meksika, Endonezya, Nijerya ve Türkiye'den oluşan MINT ülkeleri incelenmiştir. Bu ülkelerin seçilmesinin temel nedeni ilgili ülkelerin gelişmekte olan ülkeler grubunda olmasına karşın özellikle coğrafi konumlarından dolayı büyüme potansiyelinin bulunmasidir (Ersin, 2018).

\subsection{Pedroni Panel Eşbütünleşme Analizi}

Pedroni panel eşbütünleşme analizi değişkenler arasındaki uzun süreli ilişkinin varlığını test etmede kullanılmaktadır. Söz konusu analiz sürecinde sistem tarafından 7 farklı test (Panel v-Statistic, Panel rho-Statistic, Panel PP-Statistic, Panel ADF-Statistic, Group rho-Statistic, Group PP-Statistic ve Group ADF-Statistic) yapılmaktadır. Var olan bu testlerin en az 4 adedinin anlamlı olmas durumunda, değişkenler arasındaki uzun süreli ilişkiden söz edebilmek mümkündür. Testlere ait önem değerleri 0.05 rakamının altında olması durumunda ilgili testlerin anlamlı olduğu anlaşılmaktadır (Pedroni, 2004:597). Söz konusu analiz literatürde birçok farklı alanda yapılan çalışmalar için tercih edilmiştir (Dinçer vd., 2019c:49)

\subsection{Analiz Sonuçları}

Çalışmanın analizinde iki farklı aşama bulunmaktadır. İlk aşamada değişkenlerin durağanlık sınamaları yapılmıştır. İkinci aşamada ise Pedroni panel eşbütünleşme testi uygulanmıştır. Durağanlık sınamasında Levin, Lin ve Chu birim kök testi dikkate alınmış olup sonuçlara Tablo 1'de yer verilmiştir.

Tablo 1: Levin, Lin ve Chu Birim Kök Testi Sonuçları

\begin{tabular}{|c|c|}
\hline Değişkenler & Olasılık Değeri \\
\hline Doğrudan Yabancı Yatırım & 0.0227 \\
\hline Ekonomik Büyüme & 0.0011 \\
\hline
\end{tabular}

Tablo 1'den görüleceği üzere her iki değişkene ait olasılık değerleri 0.05 rakamının altındadır. $\mathrm{Bu}$ durum değişkenlerin düzeyde durağan olduğu bilgisini vermektedir. Dolayısıyla ikinci aşamada ilgili değişkenlerin düzey değerleri dikkate alınmıştır. Pedroni panel eşbütünleşme testi sonuçlarına ise Tablo 2'de yer verilmiştir.

Tablo 2: Pedroni Panel Eşbütünleşme Testi Sonuçları

\begin{tabular}{|c|c|}
\hline Testler & Olasılık Değeri \\
\hline Panel v-Statistic & 0.3638 \\
\hline Panel rho-Statistic & 0.0028 \\
\hline Panel PP-Statistic & 0.0086 \\
\hline Panel ADF-Statistic & 0.0799 \\
\hline Group rho-Statistic & 0.1186 \\
\hline Group PP-Statistic & 0.0513 \\
\hline Group ADF-Statistic & 0.2261 \\
\hline
\end{tabular}

Turkish Studies - Economics, Finance, Politics

Volume 14 Issue 3, 2019 
Tablo 2'de Pedroni panel eşbütünleşme analizine ilişkin 7 farklı test sonucu yer almaktadır. 3 adet teste (Panel v-Statistic, Group rho-Statistic ve Group ADF-Statistic) ilişkin olasılık değerleri $(0.3638,0.1186$ ve 0.2261$) 0.1$ rakamının üzerindedir. Buna karşın, diğer 4 teste (Panel rho-Statistic, Panel PP-Statistic, Panel ADF-Statistic ve Group PP-Statistic) ait olas1l1k değerleri (0.0028, 0.0086, 0.0799 ve 0.0513 ) ise 0.1 rakamının altındadır. Olasılık değerlerinin 0.1 rakamının altında olması testin istatistiki olarak anlamlı olduğu bilgisini vermektedir. Dolayısıyla, 7 adet testin 4'ünün istatistiki olarak anlamlı olması MINT ülkeleri için doğrudan yabancı yatırımlar ve ekonomik büyüme arasında uzun süreli ilişki olduğu bilgisini vermektedir.

\section{Sonuc}

Çalışmada doğrudan yabancı yatırımlarının ülkelerin ekonomik gelişmeleri üzerinde etkili olup olmadığı incelenmektedir. Bu çerçevede, Meksika, Endonezya, Nijerya ve Türkiye'den oluşan MINT ülkeleri inceleme kapsamına alınmıştır. Çalışmanın analiz sürecinde Pedroni panel eşbütünleşme analizi kullanılmıştır. Ayrıca, 1974 ve 2017 dönem aralığındaki yıllık veriler kullanılarak tarihsel bir analiz yapılması hedeflenmiştir.

Sonuç olarak, MINT ülkeleri için doğrudan yabancı yatırımlar ve ekonomik büyüme arasında uzun süreli ilişki olduğu sonucuna varılmıştır. Başka bir ifadeyle, doğrudan yabancı yatırımlarının arttırılması bahsi geçen ülkelerin ekonomik gelişmesine katkı sağlamaktadır. Bundan dolayı MINT ülkelerinde hükümetlerin doğrudan yabancı yatırımı arttırabilmek adına vergi indirimi ve lokasyon desteği gibi teşvikleri vermeleri yerinde olacaktır. Bu sayede, bahsi geçen gelişmekte olan ülkelerin gelişmiş ülke seviyesine ulaşabilmeleri daha kolay olacaktır.

Literatürde önem arz eden bu konu MINT ülkeleri üzerinde test edilerek katkı sağlanması amaçlanmıştır. Bununla birlikte gelecekteki çalışmalarda önemli olan bu konunun farklı ülke gruplarına da uygulanması yerinde olacaktır. Öte yandan, bahsi geçen bu çalışmalarda panel regresyon, Engle Granger eşbütünleşme analizi, Dumitrescu Hurlin panel nedensellik analizi gibi farklı yöntemlerin de kullanılmasının katkı sağlayacağı düşünülmektedir.

\section{KAYNAKÇA}

Adal1, Z., \& Yüksel, S. (2017). Causality relationship between foreign direct investments and economic improvement for developing economies. Marmara Journal of Economics Volume, 1(2), s. 109118.

Anuchitworawong, C., \& Thampanishvong, K. (2015). Determinants of foreign direct investment in Thailand: Does natural disaster matter?. International Journal of Disaster Risk Reduction, 14, s. 312-321.

Arun, K., \& Yıldırım, D. Ç. (2017). Effects of foreign direct investment on intellectual property, patents and R\&D. Queen Mary Journal of Intellectual Property, 7(2), s. 226-241.

Aybar, S. (2016). Determinants of Turkish outward foreign direct investment. Transnational Corporations Review, 8(1), s. 79-92.

Bekhet, H. A., \& Al-Smadi, R. W. (2015). Determinants of Jordanian foreign direct investment inflows: Bounds testing approach. Economic Modelling, 46, s. 27-35.

Borensztein, E., De Gregorio, J., \& Lee, J. W. (1998). How does foreign direct investment affect economic growth?. Journal of international Economics, 45(1), s. 115-135.

Cassidy, J. F. (2017). Japanese direct investment in China: locational determinants and characteristics. Routledge. 
Castellani, D., Meliciani, V., \& Mirra, L. (2016). The determinants of inward foreign direct investment in business services across European regions. Regional Studies, 50(4), s. 671-691.

Cergibozan, R., \& Demir, C. (2017). The determinants of foreign direct investment outflows from Turkey. In Outward Foreign Direct Investment (FDI) in Emerging Market Economies (pp. 227243). IGI Global.

Dinçer, H., Yüksel, S., \& Martínez, L. (2019). Balanced scorecard-based Analysis about European Energy Investment Policies: A hybrid hesitant fuzzy decision-making approach with Quality Function Deployment. Expert Systems with Applications, 115, s. 152-171.

Dinçer, H., Yüksel, S., Adalı, Z., \& Aydın, R. (2019a). Evaluating the Role of Research and Development and Technology Investments on Economic Development of E7 Countries. In Organizational Transformation and Managing Innovation in the Fourth Industrial Revolution (pp. 245-263). IGI Global.

Dinçer, H., Yüksel, S., Korsakienè, R., Raišienè, A. G., \& Bilan, Y. (2019b). IT2 Hybrid DecisionMaking Approach to Performance Measurement of Internationalized Firms in the Baltic States. Sustainability, 11(1), s. 296.

Dinçer, H., Yüksel, S., Pınarbaşı, F., \& Çetiner, İ. T. (2019c). Measurement of Economic and Banking Stability in Emerging Markets by Considering Income Inequality and Nonperforming Loans. In Maintaining Financial Stability in Times of Risk and Uncertainty (pp. 49-68). IGI Global.

Economou, F., Hassapis, C., Philippas, N., \& Tsionas, M. (2017). Foreign direct investment determinants in OECD and developing countries. Review of Development Economics, 21(3), s. 527-542.

Erdogan, M., \& Unver, M. (2015). Determinants of foreign direct investments: Dynamic panel data evidence. International Journal of Economics and Finance, 7(5), s. 82.

Ersin, İ. (2018). İhracata Dayalı Büyüme Hipotezinin Test Edilmesi: MINT Ülkeleri Örneği. Ekonomi, İsletme ve Maliye Araştırmaları Dergisi, 1(1), 26-38.

Iamsiraroj, S. (2016). The foreign direct investment-economic growth nexus. International Review of Economics \& Finance, 42, s. 116-133.

Iamsiraroj, S., \& Ulubaşoğlu, M. A. (2015). Foreign direct investment and economic growth: A real relationship or wishful thinking?. Economic Modelling, 51, s. 200-213.

Jimborean, R., \& Kelber, A. (2017). Foreign direct investment drivers and growth in Central and Eastern Europe in the aftermath of the 2007 global financial crisis. Comparative Economic Studies, 59(1), s. 23-54.

Jude, C., \& Silaghi, M. I. P. (2016). Employment effects of foreign direct investment: New evidence from Central and Eastern European countries. International Economics, 145, s. 32-49.

Kariuki, C. (2015). The determinants of foreign direct investment in the African Union. Journal of Economics, Business and Management, 3(3), s. 346-351.

Lucke, N., \& Eichler, S. (2016). Foreign direct investment: the role of institutional and cultural determinants. Applied Economics, 48(11), s. 935-956.

Lv, P., \& Spigarelli, F. (2016). The determinants of location choice: Chinese foreign direct investments in the European renewable energy sector. International Journal of Emerging Markets, 11(3), s. 333-356. 
Nielsen, B. B., Asmussen, C. G., \& Weatherall, C. D. (2017). The location choice of foreign direct investments: Empirical evidence and methodological challenges. Journal of World Business, 52(1), s. 62-82.

Paramati, S. R., Ummalla, M., \& Apergis, N. (2016). The effect of foreign direct investment and stock market growth on clean energy use across a panel of emerging market economies. Energy Economics, 56, s. 29-41.

Pedroni, P. (2004). Panel cointegration: asymptotic and finite sample properties of pooled time series tests with an application to the PPP hypothesis. Econometric theory, 20(3), s. 597-625.

Salahuddin, M., Alam, K., Ozturk, I., \& Sohag, K. (2018). The effects of electricity consumption, economic growth, financial development and foreign direct investment on $\mathrm{CO} 2$ emissions in Kuwait. Renewable and Sustainable Energy Reviews, 81, s. 2002-2010.

Seker, F., Ertugrul, H. M., \& Cetin, M. (2015). The impact of foreign direct investment on environmental quality: a bounds testing and causality analysis for Turkey. Renewable and Sustainable Energy Reviews, 52, s. 347-356.

Seyoum, M., Wu, R., \& Lin, J. (2015). Foreign direct investment and economic growth: The case of developing African economies. Social Indicators Research, 122(1), s. 45-64.

Solarin, S. A., \& Shahbaz, M. (2015). Natural gas consumption and economic growth: the role of foreign direct investment, capital formation and trade openness in Malaysia. Renewable and Sustainable Energy Reviews, 42, s. 835-845.

$\mathrm{Su}$, Y., \& Liu, Z. (2016). The impact of foreign direct investment and human capital on economic growth: Evidence from Chinese cities. China Economic Review, 37, s. 97-109.

Sunde, T. (2017). Foreign direct investment, exports and economic growth: ADRL and causality analysis for South Africa. Research in International Business and Finance, 41, s. 434-444.

Tang, D. (2017). The determinants of European Union (EU) foreign direct investments in the EU countries from Central and Eastern Europe during 1994-2012. Comparative Economic Research, 20(1), s. 75-99.

Tokunaga, M., \& Iwasaki, I. (2017). The Determinants of Foreign Direct Investment in Transition Economies: A Meta- analysis. The World Economy, 40(12), s. 2771-2831.

Tunay, K. B., \& Yüksel, S. (2017). The relationship between corporate governance and foreign ownership of the banks in developing countries. Contaduría y administración, 62(5), s. 16271642.

Villaverde, J., \& Maza, A. (2015). The determinants of inward foreign direct investment: Evidence from the European regions. International Business Review, 24(2), s. 209-223.

Xaypanya, P., Rangkakulnuwat, P., \& Paweenawat, S. W. (2015). The determinants of foreign direct investment in ASEAN: The first differencing panel data analysis. International Journal of Social Economics, 42(3), s. 239-250.

Young, C. (2017). Place marketing for foreign direct investment in Central and Eastern Europe. In Foreign Direct Investment and Regional Development in East Central Europe and the Former Soviet Union (pp. 141-160). Routledge.

Zengin, S., Yüksel, S., \& Kartal, M. T. (2018). Understanding the Factors that aFFect Foreign direct investment in tUrkey by Using mars method. Finansal Araştırmalar ve Çalışmalar Dergisi, 10(18), s. 1309-1123. 